\title{
Erratum to: Reliability and validity of the Nijmegen Cochlear Implant Questionnaire in Spanish
}

\author{
Isabel Sanchez-Cuadrado $\cdot$ Javier Gavilan • \\ Rosa Perez-Mora $\cdot$ Elena Muñoz $\cdot$ Luis Lassaletta
}

Published online: 10 April 2014

C) Springer-Verlag Berlin Heidelberg 2014

\section{Erratum to: Eur Arch Otorhinolaryngol}

\section{DOI 10.1007/s00405-014-2983-9}

By error, the alignment of Table 4 column heads was incorrect in the original publication of the article. The corrected Table 4 is given below.

Table 4 Validity as determined using Pearson's correlation coefficient

\begin{tabular}{llllllll}
\hline & $\begin{array}{l}\text { Basic sound } \\
\text { perception }\end{array}$ & $\begin{array}{l}\text { Advanced sound } \\
\text { perception }\end{array}$ & $\begin{array}{l}\text { Speech } \\
\text { production }\end{array}$ & Self-esteem & Activity & Social interaction & Total score \\
\hline $\begin{array}{l}\text { Bisyllables without lip-reading without masking } \\
\text { Pearson correlation }\end{array}$ & 0.063 & $\mathbf{0 . 2 4 1}$ & $\mathbf{0 . 2 4 3}$ & $\mathbf{0 . 2 3 5}$ & 0.113 & 0.172 & 0.230 \\
$\begin{array}{l}\text { Significance } \\
\text { (2-sided) }\end{array}$ & 0.602 & $\mathbf{0 . 0 4 1}$ & $\mathbf{0 . 0 3 9}$ & $\mathbf{0 . 0 4 7}$ & 0.343 & 0.148 & 0.052 \\
\hline
\end{tabular}

Values marked in bold indicate a significant correlation A $p$ value of $<0.05$ was considered statistically significant

The online version of the original article can be found under doi:10.1007/s00405-014-2983-9.

I. Sanchez-Cuadrado $(\bowtie) \cdot$ J. Gavilan · R. Perez-Mora •

E. Muñoz $\cdot$ L. Lassaletta

Department of Otolaryngology Head and Neck Surgery, Idipaz

Hospital La Paz Institute for Health Research, $\mathrm{P}^{\circ}$ Castellana,

261, 28046 Madrid, Spain

e-mail: iscuadrado@gmail.com 